\title{
Primary Cancer Cell Metastasis and Secondary Niches: Novel Cues from Metabolomics for Strategic Therapy
}

Ramalingam $\mathbf{K}^{1,2,3}$

${ }^{1}$ Department of Marine Biotechnology \& Centre for Marine Bioprospecting, Academy of Maritime Education and Training (AMET) University, Chennai, India

${ }^{2}$ Mediclone Biotech, Chennai, Tamilnadu, India

${ }^{3}$ Dr. Rai Memorial Medical (Cancer Hospital) Centre, (Ethical Committee), Teynampet, Chennai, Tamil Nadu, India

*Corresponding author: Ramalingam K, Department of Marine Biotechnology \& Centre for Marine Bioprospecting, Academy of Maritime Education and Training (AMET) University, Chennai, India, Tel: 91 - 44 26161180; E-mail: krmbiomed@gmail.com

Rec date: May 01, 2015; Acc date: May 20, 2015; Pub date: May 22, 2015

Copyright: (c) 2015 Ramalingam K, et al. This is an open-access article distributed under the terms of the Creative Commons Attribution License, which permits unrestricted use, distribution, and reproduction in any medium, provided the original author and source are credited.

Keywords: Cancer; Secondary niches

\section{Introduction}

Cancer which is considered a highly complex and heterogenous disease involves a series of genetic changes leading to multiple single nucleotide polymorphisms (SNP's) and the eventual synthesis of unlimited protein molecules through combined transcriptional and translational processes and their epigenetic expressions to transform a normal cell into a cancer cell capable of having acquired the six cardinal characteristics, as delineated by Hanahan and Weinberg [1] viz., self-sufficiency in growth signals; insensitivity to anti-growth signals; evasion of apoptosis; limitless replicative potential; sustained angiogenesis ; and tissue invasion and metastasis. Cancers irrespective of their types and organs exhibit certain common phenotypic traits and functional attributes such as uncontrolled cell division and autonomous growth; heritability and transplantability; altered cell surface; invasiveness and metastasis; dedifferentiation and loss of specialized functions; stem cells differentiation etc., [2,3]. Among all phases of cancer growth metastasis is the critical point for the following reasons. (i) Metastasis enables the cancer cells to migrate to distant sites or organs. (ii) It establishes through proliferation secondary and/tertiary population of cancer/malignant cells. (iii) It enables the survival of cancer cells in a new environment or micro environmental niche i.e, in a distant organ [4] (iv) It causes the emergence of chemo resistant and radiation resistant stem cells formation and their proliferation.

Before delving into the intricacies of metastasis of cancer cells to the distant sites or organs, it is prudent to appraise the details of metastic cells and their transportation or migration media in vivo. A relevant comparison of normal cell versus malignant one, may give an insight to the functional dynamics of cancer cells motility and invasiveness. The motility or the motile behavior of normal and cancer cells have been investigated by several authors like Ranvier, Virchow, Waldeyer, Lambert and Michael Abercrombie using tissue culture [5]. These studies have revealed not only the differences between the normal and malignant cells but enlightened the anarchic social organization of a tumour/cancer mass with cells rushing in all directions completely devoid of normal restraints, which play a vital role in the invasion of adjacent tissues. Abercrombie demonstrated that normal cells showed mutual contact inhibition of locomotion ie., when a cell bumps into another cell it stops moving. On the contrary the malignant cells do not exhibit, contact inhibition but show contact guidance ie., they move along the surfaces of normal cells and tissue planes. Adjacent normal epithelial cells are mutually adhesive and stick to each other.
Malignant cells show decreased mutual adhesiveness. These can detach from the neighbours and can penetrate tissues. They could be carried in extracellular fluid from the tumour foci into the normal tissues. The tumour cells can also gain access to blood or lymph vessels and extend inside them. The above channels of lymph and blood enable the distant dissemination of the malignant cells. The motility of cancer cells gives way to the most lethal characteristics of malignancy namely the metastasis [6].

The process of metastasis involves several stages viz., the dissemination of cells, the arrest of cells at distant sites and the survival and proliferation of cells in such sites. There are many ways in which malignant cells may reach distant site. The simplest mode of transferring malignant cells from one site to another is inter epithelial contact. Another simple mode of cell transfer is by direct invasion and then dissemination within serous cavities. The latter gives rise tumour nodules formation $[7,8]$

Ovarian cancers develop extensive ascites in the peritoneal cavity with large numbers of malignant cells which spread out all over the peritoneum. Another less common form of serous dissemination involves the spread of tumour cells from the primary cancer of ovary to the upper part of the abdomen such as stomach and/or pancreas leading to secondary tumour formation. Of all the afore mentioned ways, the most significant mode of cell dissemination is intravascular either lymphatic or haematogenous [9].

\section{Lymphatic Dissemination}

The cells shed from a primary tumour rapidly gain access to the draining lymph nodes. Though the lymph nodes act as a barrier preventing the escape of malignant cells downstream to the next lymph node group or into the blood stream via the thoracic duct, the above lymph nodes barrier and their trapping function is abolished by factors such as steroid hormones or local irradiation etc. The above barrier disruption facilitates the entry of further secondary and tertiary deposits of malignant cells all along the lymphatic chain and their final access to the blood stream [10].

Heamatogenous spread is quantitatively an inefficient mechanism. But the blood supply to a cancer is being highly disorganized and is associated with chaotic breakdown of vascular integrity. Malignant invasive cells directly enter into the blood. Some forms of cancer spread exclusively via the blood stream. Choriocarcinoma, Osteogenic sarcoma, thyroid and kidney carcinomas give rise to distant metastasis through blood stream than lymph nodes. The haematogenous spread of malignant cells depends upon the vascularity of the primary tumour 
and the secretion TAF by the malignant cells in the primary tumour. Tumors of bone always metastasize via the blood stream.

Among the extracellular components in cell-substrate interactions and cell-cell interactions, fibronectin is probably the most important. It is a high molecular weight glycoprotein present in blood plasma, extracellular matrices, basal lamina and at cell surfaces. In the quiescent normal cells it plays an important role in anchorage to substrata such as collagen. Its disappearance after malignant transformation contributes to the reduced adhesiveness and increased invasiveness of the cancer cells.

Apart from the major blood borne or haematogenous and lymphatic dissemination cancer spreads by local extension and across body cavities. In local extension, invasion occurs to the adjacent sinuses. For example a large primary breast cancer may invade into skin causing ulceration. In Lung cancer the cells can make a local extension to an adjacent spinal vertebra causing erosion of the bone and damage to the spinal cord. Spread of cancer cells across a body cavity, though unusual in most of cancers, it is an extremely important mode of spread in patients with ovarian cancer.

In this, tumour cells move to abdominal cavity and completely obliterate this area causing complications to bowel movement. Besides abdominal distension, secondary tumour growth beyond abdominal cavity may be fatal within a short period, Malignant cells dissemination may take any one or all of the four above mentioned invasion mechanisms. The prior identification of the sites of primary tumour / cancer is certainly tantamount to a prospective cure, with the currently available therapeutic interventional devices [11].

In metastatic invasion, migration of cancer cells are not random but selective i.e, in most of breast cancer patients secondary metastasis and seeding occurs in skin, bones and liver. In the case of ovarian cancer it is to the upper abdominal regions viz., to stomach and pancreas. In the case of gastro interstinal cancers, the cancer cells of similar adenocarcinomatous type occurs throughout the anterioposterior axis [12]. Our basic knowledge on human physiology and tumor cell biology might enable more rational approach to existing cancer therapy.

The secondary sites of metastasizing cancer cells have their own unique metabolic profiles. For instance the liver is the main metabolic hub for various energy derivation mechanisms involving carbohydrates proteins and lipids. Likewise the different parts of the intestine may have their own unique niches with different gaseous tensions $\left(\mathrm{O}_{2}{ }^{-} \mathrm{Co}_{2}\right)$ /quotient. The bone metabolism involves the various elements like calcium, phosphorous and enzymes etc. The secondary sites or organs have their own specific enzyme system and functioning pattern of cell organelle. Hence the therapeutic strategy remains in finding the novel cues in these secondary organs which influence the organ specific tropism of primary malignant cells to the selective sites and their further proliferation. In this context, it is of interest to note the randomness or stochastic nature of cancer cells make up in the genome level, proteome level, and metabolic level. However the selective seeding of the primary malignant cells in the secondary organs such as skin, liver, bone, peritoneum, stomach and pancreas may not occur in random manner but directional as determined by the above host tissue metabolities, enzymes, hormones, growth stimulant factors etc., and the organotropism of cancer cells. The metastasizing cancer cells though have the unlimited genomic potential to undergo proliferation; their energy potential for their continued divisions is very much limited within and require supply of constituents from the without. Research should be focused to draw more information regarding the molecular level cross talks between the host tissue cells and the primary metastasizing cancer cells. Ramalingam [4] has revealed the intersection between tumour (recapitulated embryonic cells) and target cells might involve the release of immuno suppressive factors through gain of gene signatures, in order to remain free from the hosts immunosurveillance and assaults. He has also cited Hamaoka et al. [13] who showed biochemical and functional similarities between embryonic trophoblasts secretions and the tumor cells (Recapitulated embryonic cells) secretions. The proteomic factors of host tissues or that of the invaded primary metastasizing cancer cells may be the grey area to look at so as to disrupt their interactions and/or to delimit the immune suppressors and the activation of cytotoxic immune cells.

The various metabolic profiles to look for in the host tissues include their levels of negatively charged phospholipids/cardio-lipins, platelet function, prostacyclin, protein-c function, phosphoserine level and lactic acid etc. (Figure 1) [14-16].

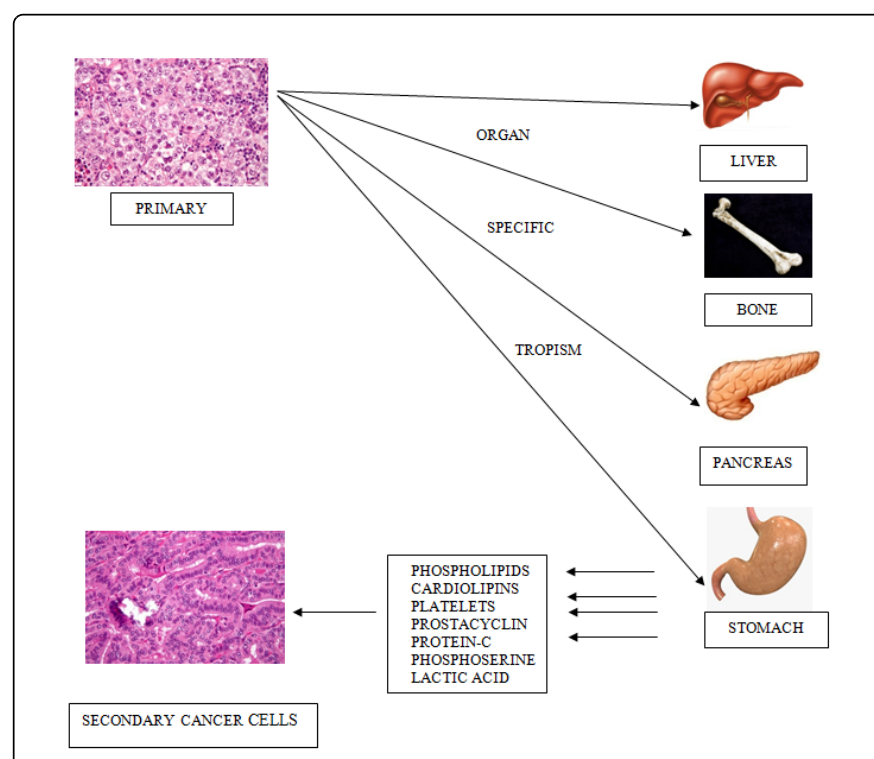

Figure 1: Metabolomic model in cancer therapeutic strategy.

In cancer treatment the principle of chemotherapy was adopted since the discovery of nitrogen mustards and antifolate drugs. The potential therapeutic value of a number compounds or drugs such as nitrogen mustard, cyclophosphamide, chlorambucil, folate analogoues viz, aminopterin and amethopterin methotrexate in causing tumor regression has been well established. Thus in cancer therapy after surgical removal of the primary tumor, the principle of chemical adjuvant therapy was established [17]. Further, the expression of receptors viz, ER, PR, HER2 neu etc. over the cancer cell surface especially in the breast cancer cells, prompted hormonal therapy in cancer treatment modality. Consequent to the realization that chemotherapy and radiation dosages have their own innate demerits of developing resistant cancer cell mutant populations, the research focus laid the emphasis on immunotherapy and the development of monoclonal antibodies come to stay put in the target oriented cancer therapy. Even these mabs (Monoclonal antibodies) and other genetic anti cancer agents such as siRNAs and mi RNAs show poor pharmacokinetic profiles as they are distributed non-specifically in the 
Citation: Ramalingam K (2015) Primary Cancer Cell Metastasis and Secondary Niches: Novel Cues from Metabolomics for Strategic Therapy. J

Page 3 of 3

body leading to systemic toxicity associated with serious side effects as cited by Hong Yeol Yoon et al. [18]. So, inspite of all these modalities of treatment, the cure for cancer has become a far cry; rather prolongation of survival time in patients has become the success story. In this context, the treatment strategies were aimed so far to target the cancer cells and the drugs were designed and tailored as "one stitch common for all". It is high time to probe and think over the surrounding niches/environment around the cancer mass which may have a plethora of metabolites acting for and against their proliferation and survival. Compensating the deficient metabolites by supply, and eliminating the injurious metabolites would be a compromising and promising metabolomic strategy towards cancer therapy.

\section{References}

1. Hanahan D, Weinberg RA (2011) Hallmarks of cancer: the next generation. Cell 144: 646-674.

2. Akhtar SS (1991) Current oncology. Matkkoff Press.

3. Ranga MM (2003) Animal Biotechnology. Agrobios (India)

4. Ramalingam K (2015) Gene Signatures and Gene Function Profiles in Cancer Manifestation- A Review. International Journal of Recent Scientific Research 6: 2 .

5. Brachet J, Mirsky AE (1961) The cell - Biochemistry, Physiology and Morphology. Academic Press.

6. Monteiro J, Fodde R (2010) Cancer stemness and metastasis: therapeutic consequences and perspectives. Eur J Cancer 46: 1198-1203.

7. Spano D, Heck C, De Antonellis P, Christofori G, Zollo M (2012) Molecular networks that regulate cancer metastasis. Semin Cancer Biol 22: 234-249.
8. Liao WT, Ye YP, Deng YJ, Bian XW, Ding YQ (2014) Metastatic cancer stem cells: from the concept to therapeutics. Am J Stem Cells 3: 46-62.

9. Mareel MM, Calman KC (1984) Invasion- Experimental and Clinical Implications. Oxford University Press.

10. Currie G, Currie A (1982) Cancer - the biology of malignant diseases. Edward Arnold, Times of Castlefield Press.

11. Fortner JG, Rhoads JE (1986) Accomplishments in Cancer Research 1986 J.B. Lippincott Company, Philadelphia.

12. Ramalingam K, Indra Alias, Muthu Meena P (2015) Cytomorphology of gastro - intestinal cancer of south india cohort - case studies report. Cytology and Histology (Sent for Publication).

13. Hamaoka T, Matsuzaki N, Itoh K, Tsuji Y, Izumi Y, et al. (1983) Humantrophoblast and tumor cell-derived immunoregulatory factor. In: Isojima S, Billington WD (eds.) Reproductive Immunology. Elsevier, Amsterdam.

14. Carreras LO, Vermylen JG (1982) "Lupus" anticoagulant and thrombosis-possible role of inhibition of prostacyclin formation. Thromb Haemost 48: $38-40$.

15. Harris EN, Chan JK, Asherson RA, Aber VR, Gharavi AE, et al. (1986) Thrombosis, recurrent fetal loss, and thrombocytopenia. Predictive value of the anticardiolipin antibody test. Arch Intern Med 146: 2153-2156.

16. Lyden TW, Ng AK, Rote NJ (1992) Modulation of phosphatidyl serine epitope expression on Benin cells during forskolin treatment. Am J Reprod Immunol 27: 24.

17. Freireich EJ (2002) Min Chiu Li: a perspective in cancer therapy. Clin Cancer Res 8: 2764-2765.

18. Yoon HY, Son S, Lee SJ, You DG, Yhee JY, et al. (2014) Glycol chitosan nanoparticles as specialized cancer therapeutic vehicles: sequential delivery of doxorubicin and Bcl-2 siRNA. Sci Rep 4: 6878. 